\title{
Clinical and dermoscopic features of a verrucous epidermal neavus
}

\section{Sara Oukarfi, Rhizlane Chaoui, Salim Gallouj, Fatima Zahra Mernissi}

\author{
Department of Dermatology CHU Hassan II, Fez, Morocco
}

Corresponding author: Dr. Sara Oukarfi, E-mail: oukarfisara@gmail.com

Sir

Epidermal nevi are defined as cutaneous hamartomas with several clinical forms. The verrucous form is the typical and most frequent form of epidermal neavus. We presented a 4 years old boy, with no particular medical history, was admitted for linear pigmented lesions of the right upper limb evolving since birth. Dermatological examination unveiled linear pigmented plaques starting at the dorsal surface of the thumb reaching to the elbow, well defined with irregular contours, with warty surface, resting on a skin hypopigmented in places (Fig. 1).

Dermoscopy revealed a cerebral aspect of the lesions, brown dots, white and brown exophytic papillary structures, and thick, adherent scales with some dot vessels.

The diagnosis was a verrucous epidermal neavus (VEN) and a CO2 laser was proposed as a treatment (Figs. 2 -4).

The dermoscopic aspects of VEN have been described in a few articles; two studies were the subject of these aspects by Carbotti et al. [1] analyzing 8 patients with VEN, and more recently Elmas and Akdeniz [2] who treated 20 cases.

Carbotti et al. [1] reported lesions of VEN showed large brown circles and the absence of cysts, pigmented networks, and globules similar to seborrheic keratoses.

Elmas and Akdeniz [2] identified new non-vascular dermoscopic aspects that had not previously been

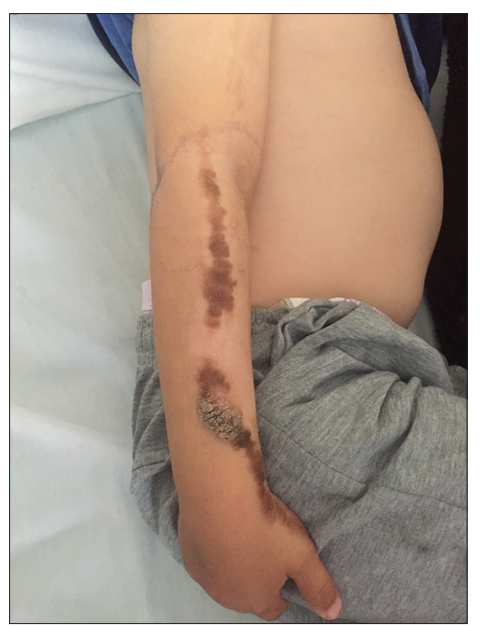

Figure 1: Clinical image of verrucous epidermal neavus.

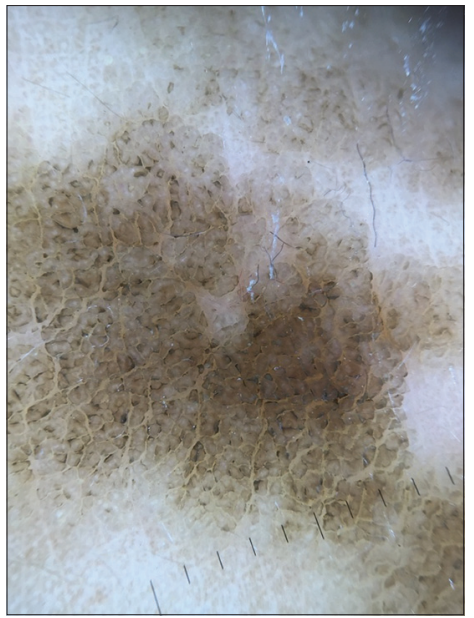

Figure 2: Thick branched brown lines and Cerebriform structures.

described for VEN. These findings are thick branched brown lines, terminal hairs, brown dots, white and 


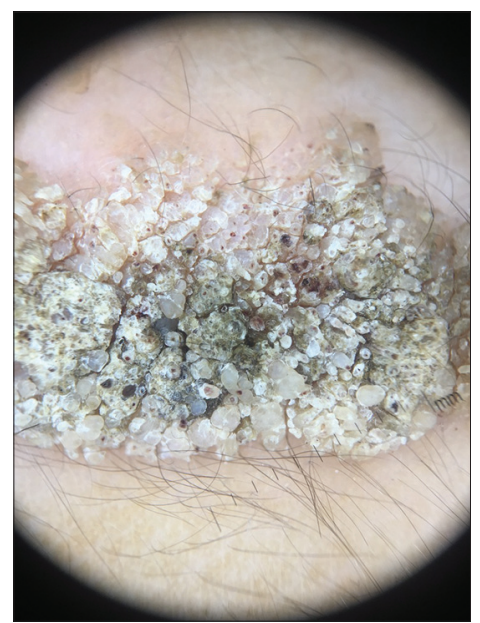

Figure 3: Thick adherent scales, dot vessels, papilliforme aspect.

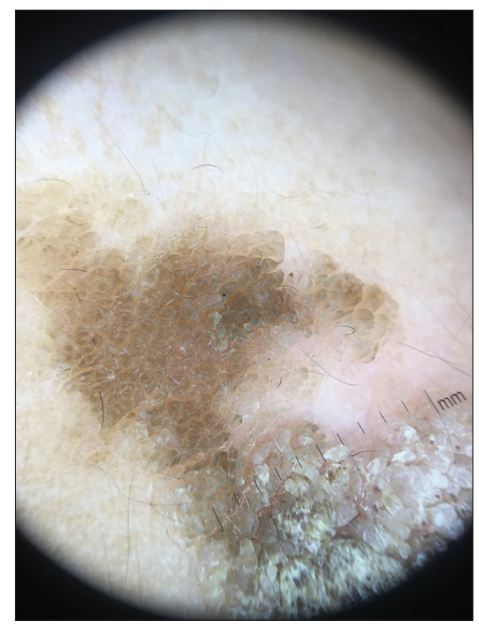

Figure 4: Cerebriform structures.

brown exophytic papillary structures, thick and adherent thick scales, thick branched lines, brown dots, brown serpiginous cerebral structures. With regard to the dermoscopic description of the vessels, the common aspect was the point vessels; the coiled, looped, serpiginous and polymorphic vessels were also present in some lesions.

In conclusion, verrucous epidermal neavuses may have dermoscopic characteristics similar to those of seborrheic keratosis and dermal nevi.

We suggest that dermoscopic examination may help in the diagnosis of VEN with decreased biopsy use. However, if there is a vascular appearance, polymorphic, which may be a malignant growth index, it would be reasonable to confirm the diagnosis by histopathology.

\section{Consent}

The examination of the patient was conducted according to the Declaration of Helsinki principles.

\section{REFERENCES}

1. Carbotti M, Coppola R, Graziano A, Verona Rinati M, Paolilli FL, et al. Dermoscopy of verrucous epidermal nevus: large brown circles as a novel feature for diagnosis. Int J Dermatol. 2016;55:653-6.

2. Elmas ÖF, Akdeniz N. Dermoscopic aspect of verrucous epidermal nevi: new findings. Turk J Med Sci. 2019;49:710-4.

Copyright by Sara Oukarfi, et al. This is an open-access article distributed under the terms of the Creative Commons Attribution License, which permits unrestricted use, distribution, and reproduction in any medium, provided the original author and source are credited.

Source of Support: Nil, Conflict of Interest: None declared. 